\title{
Fundamental thermal fluctuations in microspheres
}

\author{
Michael L. Gorodetsky \\ Faculty of Physics, Moscow State University, Moscow 119899, Russia \\ Ivan S. Grudinin \\ Department of Physics, California Institute of Technology, Pasadena, California 91125
}

\begin{abstract}
Received June 16, 2003; revised manuscript received October 6, 2003; accepted November 11, 2003
We present a theoretical analysis and the results of measurements of thermorefractive noise in microcavities. These measurements may be considered direct observations of fundamental fluctuations of temperature in solid media. Our experimentally measured noise spectra are in agreement with our theoretical model. (C) 2004 Optical Society of America

OCIS codes: $030.4070,120.6810,230.5750,160.6030$.
\end{abstract}

\section{INTRODUCTION}

The development of modern technology in many fields has led to further miniaturization of components. This makes it necessary to take into account certain fundamental physical limitations. An example of such limitations is the thermodynamical fluctuation of temperature in a small volume. Such fluctuations are transformed into wideband noise in output channels because of the temperature dependence of device parameters. The same limitations also appear frequently in experimental physics in macroscopic high-precision measurements. As was recently shown, fundamental fluctuations with the same origin limit the sensitivity of gravitational wave antennas [such as at the international Laser Interferometer Gravitational Wave Observatory ${ }^{1}$ (LIGO)], where thermal expansion, the thermal dependence of refractive indices, and Young's modulus give rise to different types of noise. $^{2-5}$ Thermorefractive fluctuations lead to phase noise in long fibers, ${ }^{6,7}$ which were used in observation of the effect for the first reported time. ${ }^{8}$

Microspheres ${ }^{9}$ are a relatively novel type of optical resonator that uniquely combine small size (from tens to thousands of micrometers) and high quality factor, as much as $Q \simeq 10^{10}$ for the so-called whispering-gallery modes $^{10}$ (WGMs). The small size of the effective volume occupied by the electromagnetic (e.m.) field of the mode makes for low thresholds of nonlinear effects such as bistability and oscillatory instability, ${ }^{11}$ which are preconditioned by Kerr's and thermal effects. The small volume makes it possible for such resonators to be used as tools for detection and measurement of thermorefractive noise. $^{12}$ Apart from its importance in the LIGO project, the measurement of thermorefractive noise can serve as an innovative experimental examination of the theory of microscopic fluctuations of temperature. Thermorefractive noise should also be taken into account in possible applications of WGM resonators such as in diode-laser stabilization.

\section{THERMOREFRACTIVE NOISE IN MICRORESONATORS}

One can understand the effect of thermorefractive noise from the well-known thermodynamic equation for the variance of temperature fluctuations $u$ in volume $V$ :

$$
\left\langle u^{2}\right\rangle=\frac{\kappa T^{2}}{\rho C V},
$$

where $T$ is the temperature of the heat bath, $\kappa$ is the Boltzmann constant, $\rho$ is density, and $C$ is specific heat capacity. By substituting $V_{\text {eff }} \simeq 10^{-9} \mathrm{~cm}^{3}$ in the following parameters for fused silica: $\rho=2.2 \mathrm{~g} / \mathrm{cm}^{3}, \quad C=6.7$ $\times 10^{6} \mathrm{erg} /(\mathrm{g} \mathrm{K})$, and the effective volume of the field of the most localized WGM in the microsphere of radius $R$ $\sim 50 \mu \mathrm{m}$, we obtain the value of the standard deviation of temperature, $\sqrt{\left\langle u^{2}\right\rangle} \simeq 30 \mu \mathrm{K}$. These temperature fluctuations, combined with the coefficient of thermal refraction $\mathrm{d} n / \mathrm{d} T=1.45 \times 10^{-5} \mathrm{~K}^{-1}$, lead to the effect of relative eigenfrequency fluctuations $\delta \omega / \omega \sim 3 \times 10^{-10}$. The estimate is comparable with the bandwidth of resonances that are achievable in microspheres. We do not consider thermoelastic noise in microspheres, as coefficient of thermal expansion $\alpha=5.5 \times 10^{-7} \mathrm{~K}^{-1}$ is sufficiently smaller than $\mathrm{d} n / \mathrm{d} T$ in fused silica. In subsequent sections we present more-rigorous analyses aimed at finding the spectral properties of this noise, taking into account the peculiar field distribution of WGMs.

Variations of refractive index $n$ in the dielectric cavity perturb the cavity's resonant frequencies. The perturbed wave equation has the form

$$
\Delta \mathbf{E}+\left(\epsilon^{0}+2 n \delta n\right) \frac{\omega^{2}}{c^{2}} \mathbf{E}=0,
$$

where $\mathbf{E}$ is the electric field strength in the cavity, $\epsilon^{0}$ $=n^{2}$ is permittivity, and $\delta n=(\mathrm{d} n / \mathrm{d} T) u$ is the variation of refractive index that is due to fluctuations of temperature $u$. If $\mathbf{E}_{0}$ is the orthonormalized field distribution of 
an eigenmode of the unperturbed cavity $\left(\int \mathbf{E}_{i} \cdot \mathbf{E}_{j}^{*} \mathrm{~d} \mathbf{r}\right.$ $=\delta_{i j}$ ) and $\omega=\omega_{0}+\delta \omega$ is the frequency shift, then after multiplication of this vector equation by complex conjugated vector $\mathbf{E}_{0} *$ and integration over the whole volume and neglecting second-order terms, we obtain

$$
\frac{\delta \omega}{\omega_{0}}=-\frac{1}{n} \int_{V}\left|\mathbf{E}_{0}{ }^{2}\right| \delta n \mathrm{~d} \mathbf{r}=-\frac{1}{n} \frac{\mathrm{d} n}{\mathrm{~d} T} \bar{u},
$$

where $\bar{u}$ is the temperature deviation averaged over the mode volume.

\section{POWER SPECTRAL DENSITY OF THERMAL FLUCTUATIONS}

To calculate the effect of fluctuations of temperature, one may use the method of fluctuational thermal sources $F(\mathbf{r}, t)$ (Refs. 2 and 3 ):

$$
\frac{\partial u}{\partial t}-D \Delta u=F(\mathbf{r}, t),
$$

where $D=\lambda * /(\rho C)$ is thermal diffusivity and $\lambda^{*}$ is thermal conductivity $\left(\lambda^{*}=1.4 \times 10^{5} \mathrm{erg} /(\mathrm{cm} \mathrm{s} \mathrm{K})\right.$ and $D$ $=9.5 \times 10^{-3} \mathrm{~cm}^{2} / \mathrm{s}$ for fused silica). This approach is analogous to the Langevin approach, which uses fluctuational forces in the equations of dynamics. It was shown before ${ }^{2,3}$ that, if the sources are properly normalized,

$$
\begin{aligned}
B_{r t}^{F} & =\left\langle F(\mathbf{r}, t) F\left(\mathbf{r}^{\prime}, t^{\prime}\right)\right\rangle \\
& =\frac{2 \kappa T^{2} D}{\rho C} \Delta \delta\left(\mathbf{r}-\mathbf{r}^{\prime}\right) \delta\left(t-t^{\prime}\right),
\end{aligned}
$$

this approach will lead to the correct results, which will satisfy the fluctuation-dissipation theorem. In particular, it was shown that thermoelastic noise is associated through the fluctuation-dissipation theorem with thermoelastic damping. It is also possible to show that thermorefractive noise is connected through the fluctuationdissipation theorem with electrocaloric losses. ${ }^{13}$

Thermodynamic fluctuations of temperature, averaged over the mode volume, may be calculated as

$$
\bar{u}=\int u(\mathbf{r}, t)\left|E_{0}(\mathbf{r})\right|^{2} \mathrm{~d} \mathbf{r}
$$

where $\int\left|\mathbf{E}_{0}\right|^{2} \mathrm{~d} \mathbf{r}=1$ and

$$
u(\mathbf{r}, t)=\int \frac{F(\mathbf{q}, \Omega)}{D q^{2}+i \Omega} \exp (i \Omega t+i \mathbf{q} \cdot \mathbf{r}) \frac{\mathrm{d} \Omega \mathrm{d} \mathbf{q}}{(2 \pi)^{4}}
$$

is the general solution of Eq. (4), where $\mathbf{q}$ is a vector in Fourier space. Spectral correlations of fluctuational forces satisfy the following condition:

$$
\begin{aligned}
B_{q \Omega}{ }^{F} & =\left\langle F\left(\mathbf{q}^{\prime}, \Omega^{\prime}\right) F^{*}(\mathbf{q}, \Omega)\right\rangle \\
& =(2 \pi)^{4} \frac{2 \kappa T^{2} D}{\rho C} q^{2} \delta\left(\mathbf{q}-\mathbf{q}^{\prime}\right) \delta\left(\Omega-\Omega^{\prime}\right) .
\end{aligned}
$$

We may now calculate the following average value: $B_{\tau}{ }^{u}=\langle\bar{u}(t) \bar{u}(t+\tau)\rangle$ (the correlation function of temperature fluctuations averaged over the mode volume) and so, from the Wiener-Hinchin theorem, the one-sided (hence additional factor of 2) power spectral density $S_{\bar{u}}(\Omega)$ of fluctuations of temperature:

$$
\begin{aligned}
S_{\bar{u}}(\Omega)= & \frac{4 \kappa T^{2} D}{\rho C} \iiint \frac{q^{2}\left|\mathbf{E}_{0}(\mathbf{r})\right|^{2}\left|\mathbf{E}_{0}\left(\mathbf{r}^{\prime}\right)\right|^{2}}{D^{2} q^{4}+\Omega^{2}} \\
& \times \exp \left[i \mathbf{q} \cdot\left(\mathbf{r}-\mathbf{r}^{\prime}\right)\right] \mathrm{d} \mathbf{r} \mathrm{d} \mathbf{r}^{\prime} \frac{\mathrm{d} \mathbf{q}}{(2 \pi)^{3}} \\
= & \frac{4 \kappa T^{2} D}{\rho C} \int \frac{q^{2}|G(\mathbf{q})|^{2}}{D^{2} q^{4}+\Omega^{2}} \frac{\mathrm{d} \mathbf{q}}{(2 \pi)^{3}},
\end{aligned}
$$

where

$$
G(\mathbf{q})=\int\left|\mathbf{E}_{0}\right|^{2} \exp (-i \mathbf{q} \cdot \mathbf{r}) \mathrm{d} \mathbf{r}
$$

is the normalized spatial spectrum of the energy distribution in the resonator. To verify this useful expression we may integrate it over all frequencies:

$$
\left\langle u^{2}\right\rangle=\int_{0}^{\infty} S_{\bar{u}}(\Omega) \frac{\mathrm{d} \Omega}{2 \pi}=\frac{\kappa T^{2}}{\rho C} \int|G(\mathbf{q})|^{2} \frac{\mathrm{d} \mathbf{q}}{(2 \pi)^{3}} .
$$

Comparing the final expression with Eq. (1), we deduce that

$$
V_{\text {eff }}^{-1}=\int|G(\mathbf{q})|^{2} \frac{\mathrm{d} \mathbf{q}}{(2 \pi)^{3}}=\int|\mathbf{E}(\mathbf{r})|^{4} \mathrm{~d} \mathbf{r} .
$$

The same general expression for the effective volume of the mode in the microresonator appears in the analysis of nonlinearity ${ }^{11}$ and scattering ${ }^{14}$ in microspheres.

It is important to note that we obtained the above expressions by ignoring boundary conditions. If the field is concentrated near a thermally isolated surface, as it is for microspheres, Eq. (8) should be modified by substitution of $\left[\delta\left(\mathbf{q}_{\perp}-\mathbf{q}_{\perp}{ }^{\prime}\right)+\delta\left(\mathbf{q}_{\perp}+\mathbf{q}_{\perp}{ }^{\prime}\right)\right] \delta\left(\mathbf{q}_{\|}-\mathbf{q}_{\|}{ }^{\prime}\right)$ for $\delta(\mathbf{q}$ - $\mathbf{q}^{\prime}$ ), where $\mathbf{q}_{\perp}$ is the component of the wave vector of fluctuations normal to the surface and $\mathbf{q}_{\|}$are components that are parallel to it.

In the analysis above, the medium was considered infinite (the volume of field localization is significantly smaller than the size of a device). A discrete spectrum of thermal waves should be considered for more-accurate calculations, especially at low frequencies:

$$
F(\mathbf{r}, t)=\int \sum F_{\nu}(\Omega) \Phi_{\nu}(\mathbf{r}) \exp (i \Omega t) \frac{\mathrm{d} \Omega}{2 \pi} .
$$

Now, as before, we may calculate the correlation function of relative frequency fluctuations and power spectral density:

$$
S_{\bar{u}}(\Omega)=\frac{4 \kappa T^{2} D}{\rho C} \sum_{\nu} \frac{q_{\nu}{ }^{2}\left|G_{\nu}\right|^{2}}{D^{2} q_{\nu}{ }^{4}+\Omega^{2}},
$$

where $G_{\nu}$ are the coefficients of field's intensity decomposition into normal thermal waves $\Phi_{\nu}(\mathbf{r})$ of the finite medium.

Applying the expressions obtained to the microsphere leads to the following expression for the power spectral density of relative frequency fluctuations: 


$$
\begin{aligned}
S_{\delta \omega / \omega}(\Omega)= & S_{\bar{u}}\left(\frac{\mathrm{d} n}{\mathrm{~d} T}\right)^{2} \\
= & \frac{\kappa T^{2} \sqrt{l}}{\pi^{3 / 2} n^{2} R^{2} \sqrt{\lambda^{*} \rho C \Omega}}\left(\frac{\mathrm{d} n}{\mathrm{~d} T}\right)^{2} \\
& \times \frac{1}{\left(1-b^{2} / d^{2}\right)^{1 / 2}} \frac{1}{\left[1+\left(\Omega \tau_{b}\right)^{3 / 4}\right]^{2}},
\end{aligned}
$$

where $l, m, b, d$, and $\tau_{b}$ are parameters that are determined by the mode in the microsphere (see Appendix A). This equation was experimentally verified in the study reported here.

It is essential to note that, in a finite body, $S_{\bar{u}}(\Omega)$, unlike in Eq. (15), remains limited at zero frequency. However, instead of the sum in expression (A15) below, we used Eq. (15), obtained from the continuous spectrum, to evaluate the results of the measurements. There were important reasons for doing this. First, we used too many approximations when we were obtaining the sum. These are crude approximations, especially for lower frequencies. However, it is shown in Appendix A and confirmed by numerical calculations that the sum in expression (A15) and the integral in expression (A5) lead to the same asymptotic dependence at high frequencies. Second, microspheres during fabrication are formed on short fused-silica stems, which conduct heat and couple microspheres to heat baths. The thermal exchange with the atmosphere owing to convection is also not negligible. Therefore microspheres are not thermally isolated. Third, other parasitic noise effects dominate at low frequencies. Throughout the experiment we considered this phenomenon only at frequencies $\Omega>1 / \tau_{R}=D / R^{2}$ $\sim 400 c^{-1}$ for $(R=50 \mu \mathrm{m})$.

\section{EXPERIMENTAL SETUP}

The idea of measuring thermorefractive noise in optical microspheres is quite simple (see Fig. 1). If one tunes the measuring laser's frequency to the slope of the resonance curve of a WGM, that is, to the range where the amplitude of the signal being measured depends strongly on frequency, then the trembling of the resonator's eigenfrequency is transformed into fluctuations of the intensity of the output radiation. These intensity fluctuations are then recorded and further processed.

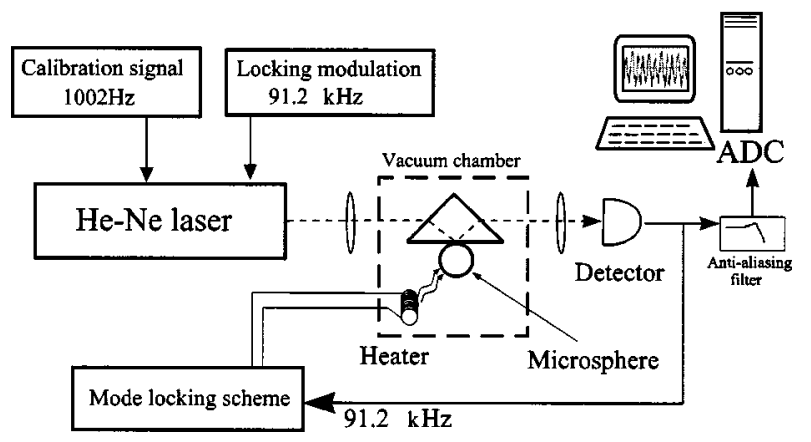

Fig. 1. Schematic of the experimental setup for measurement of noise spectra in microspheres: ADC, analog-to-digital converter.
To measure thermorefractive noise we used small $2 R$ $\simeq 80-260 \mu \mathrm{m}$ fused-silica microspheres with optical quality factors $Q \simeq 10^{9}$ manufactured with a hydrogen miniburner. To prevent degradation of the $Q$ factor owing to adsorption of atmospheric water ${ }^{10}$ we placed microspheres after fabrication in an atmosphere of dry, clean nitrogen into a special chamber where all measurements were conducted.

WGMs in microspheres were excited with a prism coupler by use of a He-Ne laser (wavelength, $\lambda=0.63 \mu \mathrm{m}$ ) with a piezo-driven front mirror, as described in Ref. 15. The laser frequency could be tuned within a range of approximately $0.8 \mathrm{GHz}$. The output power of the laser (540 $\mu \mathrm{W})$ was reduced to $45 \mu \mathrm{W}$ when the laser passed into the chamber. The reduction of power was necessary to weaken thermal and Kerr nonlinearities ${ }^{11}$ and was carried out with neutral-density filters. The frequency of the laser was locked at the slope of the resonance curve. This was done with a miniature spiral heater placed near the resonator, which actively stabilized the temperature of the microsphere, hence stabilizing the microsphere's eigenfrequency. To provide the feedback loop we used a weak signal at $91.2 \mathrm{kHz}$ to modulate the laser frequency. This frequency was chosen to coincide with one of the acoustic resonances of the front mirror to facilitate laser frequency modulation. This signal was demodulated by the WGM, and an error signal was used to correct the current that feeds the heater. The thermal response time for this locking scheme was estimated to be 4-10 ms. When the WGM is locked to the laser, the output of the microsphere is the thermorefractive amplitude noise. The output from the resonator was registered by a Thorlabs PDA500 amplified GaAsP photodetector with 40-dB gain in amplitude and $f_{d}=45 \mathrm{kHz}$ bandwidth at this gain setting.

The signal from detector was digitized with an analogto-digital converter (ICP DAS PCI-1802L computer board) with a digitization speed of $333 \mathrm{ksamples} / \mathrm{s}$ and a sample resolution of $12 \mathrm{bits} / \mathrm{s}$. Continuous sets of 6,553,600 (19.66-s) points were recorded in each measurement session for further processing. To estimate the noise frequency spectrum we subdivided the record into many equal time intervals. Power spectral densities were calculated with a fast Fourier transform algorithm for each interval and then averaged over the entire set of intervals within each session. We used 100 intervals with 65,536 $=2^{16}$ points in each interval to produce an estimate of spectra with a fine resolution of $\approx 5 \mathrm{~Hz}$. To prevent the known effect of frequency aliasing that is caused by the finite sampling rate, the frequency band of the signal incoming from the detector was limited by a Butterworth filter of eighth order. At a stop-band frequency of 166 $\mathrm{kHz}$ (the Nyquist frequency in our case) this filter attenuates the signal to $-48 \mathrm{~dB}$. A first-order RC-filter with a time constant of $\tau \simeq 2 \mathrm{~s}$ was used to get rid of the constant offset.

\section{A. Calibration of Spectra}

To obtain absolute values of spectral densities for measured spectra we have developed and used a method of calibration based on laser frequency modulation. In this method, a weak sinusoidal voltage of known amplitude 
and frequency is admixed with the output of the piezo actuator of a laser's mirror, thereby producing weak laser frequency modulation. Because the measured spectra represent relative frequency fluctuation, this additional sinusoidal modulation of laser frequency results in a narrow peak in the frequency spectrum. If the amplitude of the frequency modulation is known, it is possible to find the value of spectral density that corresponds to the spike in the spectrum of relative frequency fluctuations, i.e., in the thermorefractive noise spectrum.

To find the coefficient of transformation of voltage to laser frequency modulation and to measure the optical quality factors of the resonators, we calibrated the piezo actuator by using following technique:

The laser radiation that we used to excite the WGMs was phase modulated at a frequency that was higher than the half-width at half-maximum frequency of the modes, which added the frequency sidebands to the laser radiation. The frequency of the laser was modulated additionally by the sawtoothed voltage that provided frequency scanning and allowed us to observe the sidebands and the carrier on the oscilloscope. Two sidebands and the carrier could be observed because they excited the microsphere as the laser frequency was scanned.

These sidebands produce frequency scale for calibration of the piezo actuator and for measurement of the $Q$ factor from the widths of resonant curves. The coefficient of the piezo actuator was $\partial f / \partial v=(3.63$ $\pm 0.1)[\mathrm{MHz} / \mathrm{V}]$.

If the amplitude of frequency modulation produced by the piezo actuator is known, the amplitude of the peak that it produces in the calculated power spectral density can be used for the absolute calibration of the spectral density of thermorefractive noise (see Appendix B).

To produce a calibration peak we applied $5-15-\mathrm{mV}$ modulation at 172.9 or $1002 \mathrm{~Hz}$ (preferred frequency at later stages of experiment) to the piezo actuator of a laser mirror, which resulted in a relative frequency modulation of $P=\delta \omega / \omega=(4-11) \times 10^{-11}$. These frequencies were chosen to match those of bins of discrete Fourier transforms of 65,536 points (namely, $1001.99=197 F_{s} / 65,536$ and $\left.172.93=34 F_{s} / 65,536\right)$. The higher frequency of 1 $\mathrm{kHz}$ was chosen such that the calibration peak was separated from low-frequency setup noises in the frequency spectrum. It is important to note that the calibration method described does not require knowledge of the quality factor of the microsphere as well as of the transformation coefficients and gains in electrical tracts.

After the amplitude calibration was performed, we made an additional correction to compensate for frequency filters in the detector by dividing the resultant spectrum by $1 /\left[1+\left(f / f_{d}\right)^{2}\right]^{1 / 2}$, the frequency response of the detector. The frequency response of an antialiasing filter was also compensated for. To do this, we measured and approximated it with a polynomial of seventh order so the compensation procedure could be carried out by point-to-point multiplication.

\section{B. Background Noises in the Measurements}

In this research the background noises were represented by the electrical interference (generally $50 \mathrm{~Hz}$ and harmonics), acoustic and seismic noises, electromagnetic in- duction noises of devices, and also amplitude and frequency noises inherited from the laser. The frequency noise of the laser turned out to be the most essential influence on the quantity being investigated. We measured this noise together with all other technical noises of the setup. We used the same technique as for measurements of thermorefractive noise measurements; the only difference was that now relatively larger microspheres with diameters of $481,570,508,588,894$, and $619 \mu \mathrm{m}$ and modes with larger $l-m$ numbers of the order of 100 and above were used. In these modes, thermorefractive noise appears to be small enough for laser noise to dominate. Measurements of the laser noise for all the aforementioned microspheres coincided well enough. The microspheres operated as the frequency discriminators in this case.

Calibration of laser noise was carried out in the same way as was done for thermorefractive noise calibration: with the use of a calibrating peak. In measurements of the technical noise spectra a calibrating spike at a frequency of $172 \mathrm{~Hz}$ was used.

Special measures were undertaken to weaken electromagnetic hindrances in the setup. We applied appropriate signal shielding; moreover, we shielded the analog-todigital conversion board inside the computer, which resulted in a decrease in the setup noise of more than an order of magnitude. The dynamic range of the digitization system exceeded $90 \mathrm{~dB}$ (with averaging over many spectra into account).

\section{Identification of WGMs and Computer Processing}

On completion of recording we proceeded to identify the WGM indices. Knowledge of the modes' indices allows one to calculate the effective volume occupied by the electromagnetic field of the mode, as well as the theoretical energy distribution within the volume and therefore the theoretical spectral density. To perform the identification we used a digital camera to record the speckled image of the mode. (See Fig. 2 as an example of such an image). The speckled image was called forth by scattering of the mode's e.m. field at the residual molecular inhomogeneities on the surface of the microsphere. ${ }^{10}$ The approximation formulas were derived to link the microsphere's radius with index $l$ and the angular half-width of the WGM's belt with $l$ and $l-m$ (see Appendix C).

On being recorded, the images were magnified and processed with a custom-made computer program for graphic identification of the WGMs. The belt's width $(l-m$ value), simulating the sphere size, the slope, and tilt of the belt, could be controlled interactively, so the theoretical framework projection of the mode could be made to coincide with the photographic projection of the real mode. This allowed us to obtain the parameters of the mode. The error in the estimate of the $l-m$ value is proportional to $(l-m+1 / 2)^{1 / 2}$ i.e., the width of the mode belt, but is strongly influenced by the quality of the pictures obtained. Modes with $l-m \leqslant 3$ can usually be precisely recognized, whereas for $3<l-m<6$ the error in obtaining the indices approaches 1 . Index $q$ of the mode can in principle be found from the optimal coupling angle of incidence of the pump beam. ${ }^{10}$ However, we did not attempt to do this in the present research. We merely tried 


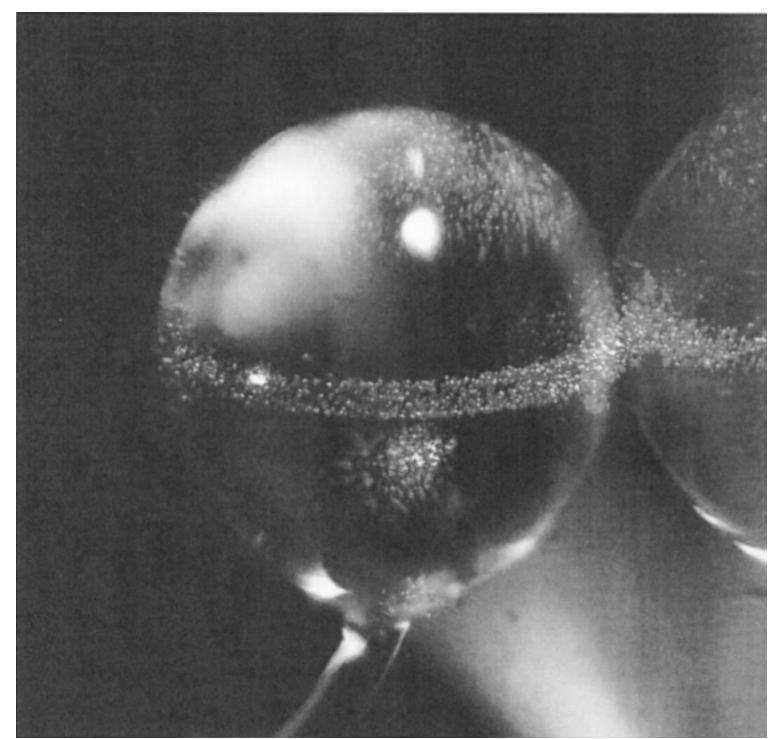

Fig. 2. Resonator of 570- $\mu$ m diameter with $l-m \simeq 13$ modes (magnification $88 \times$ ) visible as a result of surface scattering on residual inhomogeneities. At the right is a reflection of the microsphere in the coupling prism.

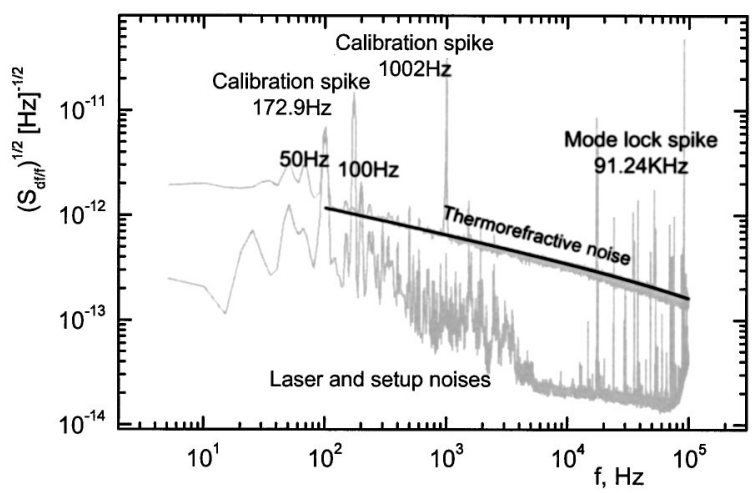

Fig. 3. Thermorefractive noise in a microsphere (138 \pm 8$) \mu \mathrm{m}$ in diameter; $l-m=4$. Lower curve, setup and laser noise, measured in a large, $894-\mu \mathrm{m}$ sphere. The straight line is a theoretical estimate.

to excite and analyze the modes with smaller $q$ indices, which are usually characterized by higher $Q$ and better coupling.

\section{THERMOREFRACTIVE NOISE SPECTRA}

Figure 3 presents a typical-high resolution spectrum of measured noise in a microsphere $138 \mu \mathrm{m}$ in diameter and the spectrum of technical noise in a large $894-\mu \mathrm{m}$ sphere measured as described in Appendix B. The black curve over the experimental curve is a theoretical curve for the spectrum calculated from the recognized value of $l-m$ $=4 \pm 1$. Good agreement of experimental data with theory for frequencies of $>100 \mathrm{~Hz}$ is clearly observable. It can also be seen that the level of combined technical noises, which include frequency and power fluctuations of the laser and electrical noises in circuits, is nearly $20 \mathrm{~dB}$ lower than the observed effect, except for isolated spikes. Therefore technical noises do not prevent experimental testing of a thermorefractive model.
Calibrating spikes at $1002 \mathrm{~Hz}$ for the smaller sphere and at $172.93 \mathrm{~Hz}$ for the larger sphere can be seen in the figure. At low frequencies, peaks of technical noise at ac, $50 \mathrm{~Hz}$-harmonics dominate. Additional sharp spikes can be seen at frequencies of the order of $20 \mathrm{kHz}$, which were identified as acoustical resonances of laser mirrors responding to acoustical noise produced by laboratory equipment. One of these resonances, at $92 \mathrm{kHz}$, was used in a feedback loop for active mode frequency stabilization (see Appendix A). These spikes, except the specially excited one at $92 \mathrm{kHz}$, are relatively small and narrow $(\sim 20 \mathrm{~Hz})$ and could be seen only because of the high resolution of our measurements $(\sim 5 \mathrm{~Hz}$ in a range of $10-10^{5} \mathrm{~Hz}$ ). They do not affect the result, especially if additional averaging at high frequencies is applied. Figure 4 depicts results of measurement of calibrated spectra of relative frequency fluctuations in four microspheres for six modes. The sizes of the microspheres and the estimated mode parameters are given in the figure.

Data for these graphics were obtained from the data presented in Fig. 3 by use of uniform averaging on a log scale according to the following algorithm: If $f_{\text {min }}\left(f_{\max } / f_{\min }\right)^{j / N}<f_{i}<f_{\min }\left(f_{\max } / f_{\min }\right)^{(j+1) / N}$, then, if $n_{j}$ $>0, f_{j}=\left(1 / n_{j}\right) \Sigma f_{i} ; S_{j}=\left(1 / n_{j}\right) \Sigma S_{i}$, where $N$ is the number of equidistant bins $(N=200$ for the figure shown) on a log scale, $n_{j}$ is the number of points found in $\operatorname{bin} j=0 \ldots N-1, f_{\min }=10 \mathrm{~Hz}$, and $f_{\max }=10^{5} \mathrm{~Hz}$. This approach allows one to estimate better the real spectra in higher frequencies while preserving frequency resolution at low frequencies. To make the figure less bulky, we digitally filtered out calibration peaks during processing.

\section{DISCUSSION}

We conclude that the results of measurements of frequency noises in microspheres confirm the theoretical model of thermorefractive fluctuations. This is true for both frequency dependence and dependence on mode parameters $l$ and $l-m$. The discrepancy is less than $30 \%$ in the frequency range $3 \times 10^{2}-10^{5} \mathrm{~Hz}\left(\Omega>1 / \tau_{R}\right)$, where the model and the approximations used are valid and are free of installation noises. For frequencies above $1 \mathrm{kHz}$ the differences are within the limits of calibration

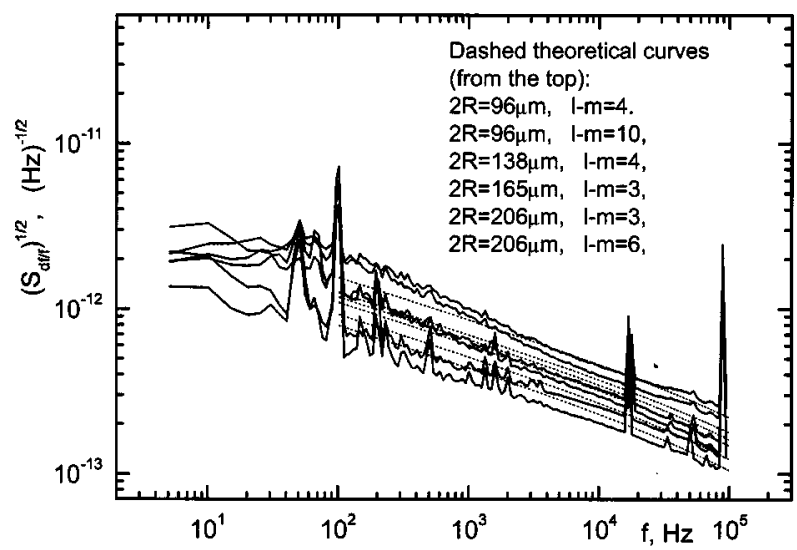

Fig. 4. Thermorefractive noise in four microspheres for six different modes. Dotted lines, theoretical curves obtained for recognized modes' parameters. Larger noise corresponds to smaller sizes of microspheres and smaller $l-m$ values. 
error and WGM identification error. Such close agreement was obtained in many resonators of average size, $120-250 \mu \mathrm{m}$ in diameter. In very small resonators, at frequencies lower than $1 \mathrm{kHz}$, the noise was as much as two times greater and the dependence on frequency was more pronounced. In large resonators at lower frequencies the noise seemed to be smaller. However, as was noted, the theoretical model described below may be not valid when the frequency of noise is comparable to the inverted time of temperature relaxation of the whole microsphere via the microsphere stem $\left(R_{s} \sim 20 \mu \mathrm{m}\right)$. The influence of the stem for smaller spheres in the ratio $R_{s} / R$ is larger. This result looks to be compatible with the observed systematic deviations at low frequencies of experimental curves from the theoretical curves obtained for thermally nonisolated spheres. Other kinds of thermal noise, such as thermoelastic ${ }^{2}$ noise and thermal fluctuations of effective eccentricity of microspheres that lead to additional frequency fluctuations proportional to $l-m$, can also contribute to the effect. Additionally, there are other influential mechanisms such as convective thermal relaxation and the influence of a WGM locking scheme, that can modify spectra.

It is worth noting that from the displayed spectra it is possible to calculate the spectra of microscopic fluctuations of temperature in the volume occupied by the e.m. field of a WGM. Using the known relation

$$
\frac{\delta \omega}{\omega}=-\frac{1}{n} \frac{\mathrm{d} n}{\mathrm{~d} T} u,
$$

one can rescale the spectra to a factor of $1 /[(1 / n)$ $\times(\partial n / \partial T)]=10^{5} \mathrm{~K}$ for fused silica. For instance, the mode of the $138-\mu \mathrm{m}$ resonator has an effective volume of the order of $4,3 \times 10^{-9} \mathrm{~cm}^{3}$, which is 4 orders of magnitude smaller than in former measurements of thermally induced phase noise in long fibers. ${ }^{8}$

\section{APPENDIX A: CALCULATION OF THERMOREFRACTIVE NOISE IN MICROSPHERES}

To simplify the analysis we perform the calculations below only for the fundamental WGM TE $\mathrm{TE}_{l l 1}$, which has the smallest volume of localization. The result in principle may be extended to account for $\mathrm{TE}_{l m q}$ modes with $q, l$ $-m \sim 1$. The field distribution of this mode may be approximated as follows:

$$
\begin{aligned}
\mathbf{E}(r, \theta, \phi) \simeq & \mathbf{E}_{\theta}(r, \theta, \phi) \frac{n l^{1 / 4}}{\sqrt{n^{2}-1} R^{3 / 2} \pi^{3 / 4}} \\
\simeq & \mathbf{i}_{\theta} \exp \left(-l \cos ^{2} \theta / 2+i l \phi\right) \\
& \times \begin{cases}j_{l}(k n r) / j_{l}(k n R) & r \leqslant R \\
\exp [-\gamma(r-R)] & r>R\end{cases} \\
k n R \simeq & +\frac{1 / 2+1.8558(l+1 / 2)^{1 / 3}}{n} \\
& -\frac{n}{\sqrt{n^{2}-1}},
\end{aligned}
$$

where $k=2 \pi / \lambda$ is a wave number in vacuum. However, even this approximation is too complex for analytical evaluation, so below we shall use the following Gaussian approximation of radial dependence:

$$
\begin{aligned}
\hat{\mathbf{e}}_{\theta}(r, \theta, \phi) \simeq & \frac{1}{\pi \sqrt{2 b d R_{0}}} \\
& \quad \times \exp \left[-\frac{\left(r-R_{0}\right)^{2}}{2 b^{2}}-\frac{r^{2} \cos ^{2} \theta}{2 d^{2}}+i l \phi\right], \\
k n R_{0} \simeq & l+1 / 2+0.71(l+1 / 2)^{1 / 3} \\
d \simeq & R_{0} l^{-1 / 2}, \\
b \simeq & \frac{1}{k n}\left[\frac{j_{l}\left(k n R_{0}\right)}{j_{l}{ }^{\prime \prime}\left(k n R_{0}\right)}\right]^{1 / 2} \simeq 0.84 R_{0} l^{-2 / 3} .
\end{aligned}
$$

This approximation describes rather adequately the distribution of optical energy inside the resonator and allows one to calculate Fourier integrals. Moreover, as will be seen below, because of the small depth of the field (parameter $b$ ), radial distribution has practically no influence on the frequency fluctuations at frequencies of interest:

$$
\begin{aligned}
|G(\mathbf{q})|= & \mid \frac{1}{2 \pi^{2} b d R_{0}} \int_{0}^{R} \int_{0}^{2 \pi} \int_{0}^{\pi} \exp \left[-\frac{r^{2} \cos ^{2} \theta}{d^{2}}\right. \\
& \left.-\frac{\left(r-R_{0}\right)^{2}}{b^{2}}\right] \exp \{i q r[\cos \theta \cos \vartheta \\
& +\sin \theta \sin \vartheta \cos (\phi-\varphi)]\} \sin \theta \mathrm{d} \theta \mathrm{d} \phi r^{2} \mathrm{~d} r \mid \\
\simeq & \exp \left[-\frac{(q d \cos \vartheta)^{2}}{4}\right] \exp \left[-\frac{(q b \sin \theta)^{2}}{4}\right] \\
& \times\left(\frac{1}{\pi q R_{0} \sin \vartheta}\right)^{1 / 2} .
\end{aligned}
$$

To obtain this result we used several approximations while we calculated the integrals. We made the first integration over angle $\theta$, taking into account that the mode distribution is narrow and hence that $\sin (\theta) \simeq 1$. The second integral, over $\phi$, led to a Bessel function of the first order, which was approximated as a spherical wave. Finally, we found the integral over $r$, considering only fastvarying functions. We also used other physical conditions to enable us to neglect small terms: $\left(R-R_{0}\right)$ $\ll R, j(k n R) \ll j\left(k n R_{0}\right)$, and $\cos (q R) \simeq 1$ that are due to boundary conditions for thermal waves. For $q \rightarrow 0$ the last term in expression (A3) should be equal to 1 and the final expression is incorrect because of approximations used for $J_{0}$. However, this case is not interesting for us. To correct the situation and to obtain a better approximation one may formally add 1 to the denominator.

Using Eq. (9) and keeping in mind the additional factor of 2 that is due to boundary conditions, we obtain 


$$
\begin{aligned}
& S_{\bar{u}}=\frac{8 \kappa T^{2} D}{\rho C} \int \frac{q^{2}|G(\mathbf{q})|^{2}}{a^{4} q^{4}+\Omega^{2}} \frac{\mathrm{d} \mathbf{q}}{(2 \pi)^{3}} \\
& \simeq \frac{2 \kappa T^{2} D}{\rho C \pi^{3} R} \int_{0}^{\infty} \int_{0}^{\pi} \exp \left[-\frac{(q d \cos \vartheta)^{2}}{2}\right] \\
& \times \exp \left[-\frac{(q b \sin \theta)^{2}}{2}\right] \frac{q^{3} \mathrm{~d} \vartheta \mathrm{d} q}{D^{2} q^{4}+\Omega^{2}}, \\
& \lim _{x \rightarrow \infty} \int_{0}^{\pi} \exp \left[-x^{2} \cos ^{2}(\vartheta)\right] \mathrm{d} \vartheta \simeq \frac{\sqrt{\pi}}{x} \\
& S_{\delta \omega / \omega}(\Omega) \simeq \frac{\kappa T^{2} D}{\pi^{5 / 2} n^{2} \rho C R} \frac{2}{\sqrt{d^{2}-b^{2}}}\left(\frac{\mathrm{d} n}{\mathrm{~d} T}\right)^{2} \\
& \times \int_{0}^{\infty} \frac{q^{2} \exp \left(-q^{2} b^{2} / 2\right)}{D^{2} q^{4}+\Omega^{2}} \frac{\mathrm{d} q}{2 \pi}, \\
& \int_{0}^{\infty} \frac{q^{2} \exp \left(-q^{2} b^{2} / 2\right)}{D^{2} q^{4}+\Omega^{2}} \frac{\mathrm{d} q}{2 \pi} \\
& \simeq \frac{\sqrt{2}}{4 D^{3 / 2} \sqrt{\Omega}} \frac{1}{\left(1+\left(\Omega \tau_{b}\right)^{3 / 4}\right)^{2}} .
\end{aligned}
$$

This integral can be expressed through special Lommel functions; however, an approximation is used here, which works well for $\Omega \tau_{b}<1$, where $\tau_{b}=(\pi / 4)^{1 / 3} b^{2} / D$. Indeed, neglecting the first term in the denominator, we obtain a high-frequency approximation with dependence $\Omega^{-2}$, and, neglecting the exponential approximation for lower frequencies, we obtain the dependence on $\Omega^{-1 / 2}$.

Finally,

$$
\begin{aligned}
S_{\delta \omega / \omega}(\Omega)= & \left(\frac{\mathrm{d} n}{\mathrm{~d} T}\right)^{2} \frac{\kappa T^{2} \sqrt{l}}{\pi^{3 / 2} n^{2} R^{2} \sqrt{\lambda^{*} \rho C \Omega}} \\
& \times \frac{1}{\left(1-b^{2} / d^{2}\right)^{1 / 2}} \frac{1}{\left[1+\left(\Omega \tau_{b}\right)^{3 / 4}\right]^{2}} .
\end{aligned}
$$

Numerical analysis, which is omitted here, shows that, for the modes with $l \neq m$, the power spectral density of the fluctuations is proportional to $[2(l-m)+1]^{1 / 2}$. This dependence is also confirmed by the fact that the azimuthal widths of the modes, and hence their effective volume, have the same dependence.

To account for the finite sizes of microspheres, one should perform calculations with Eq. (14). As at room temperature the power radiated from the surface (Stephan-Boltzmann law) is much lower than the heat exchange that is to thermal conductivity (Fourier law), the following simplified boundary condition is used:

$$
\left.\frac{\partial u(r, \theta, \phi, t)}{\partial r}\right|_{r=R}=0
$$

$$
\begin{aligned}
\Phi_{L, M, N}= & C_{L, M, N j L}\left(q_{L N} r\right) P_{L}^{M}(\cos \theta) \\
& \times\left\{\begin{array}{l}
\cos (M \phi) \\
\sin (M \phi)
\end{array}\right. \\
C_{L, M, N}{ }^{2}= & \frac{2 L+1}{\pi\left(1+\delta_{0 M}\right)} \frac{(\mathrm{A} 9)}{(L+M) !} \\
& \times \frac{\xi_{L, N}^{2}}{R^{3}\left[\xi_{L N}^{2}-L(L+1)\right] j_{L}^{2}\left(\xi_{L N}\right)} \\
G_{L, N}= & \frac{C_{L, 0, N} \int}{\pi b d R_{0}} \int \quad \exp \left[-\frac{\left(r-R_{0}\right)^{2}}{b^{2}}\right. \\
& \left.-\frac{r^{2} \cos ^{2} \theta}{d^{2}}\right] j_{L}\left(q_{L N} r\right) \\
& \times P_{L}\left(\cos ^{2} \theta\right) r^{2} \mathrm{~d} r \sin \theta \mathrm{d} \theta .
\end{aligned}
$$

Although only functions with $M=0$ lead to nonzero integrals, it is appropriate to note that functions with $M$ $=2 l$ can also be taken into account: They lead to the coupling between counterpropagating modes in the sphere and to mode splitting.

To estimate this integral for small values of $\cos \theta \simeq \psi$ $=\theta-\pi / 2$ near the equator of a microsphere, where the e.m. field is concentrated, one may use the following approximation for the Legendre polynomial $L$ $>0\left[P_{0}(\cos \theta)=1\right]$ :

$$
P_{L}(\cos \theta) \simeq \sqrt{\frac{2}{\pi L}}\left(1-\frac{1}{4 L}\right) \cos \left[\left(L+\frac{1}{2}\right) \psi+\frac{L \pi}{2}\right]
$$

and, for the spherical Bessel functions:

$$
\begin{gathered}
j_{L}(z)=\frac{1}{z} \sin (z-L \pi / 2), \\
\xi_{L N}=q_{L N} R \simeq \frac{\pi(2 N+L-1)}{2}, \\
j_{L}\left(\xi_{L N}\right) \simeq \frac{(-1)^{N-1}}{\xi_{L N}}, \quad L=2 K .
\end{gathered}
$$

It is the crudeness of the approximation for the roots of the derivatives of spherical Bessel functions that limits the applicability of the final sum that we obtain below. In this way, the calculations below may be considered to be only illustrations:

$$
\begin{aligned}
\left|G_{L, N}\right|^{2} & \simeq \frac{2 \mathcal{R}(L, N)}{\pi^{2} R^{3}} \exp \left[-\frac{(L+1 / 2)^{2} d^{2}}{2 R_{0}^{2}}-\frac{\xi_{L N}{ }^{2} b^{2}}{2 R_{0}{ }^{2}}\right] ; \\
\mathcal{R}(L, N) & =\frac{(1-1 / 4 L)^{2}(1+1 / 2 L)}{1-L(L+1) / \xi_{L N}{ }^{2}}, \quad L>0, \\
\mathcal{R}(0, N) & =\pi / 4 .
\end{aligned}
$$

To calculate these coefficients we used the approximation $q_{L N}\left(R-R_{0}\right) \ll 1$. And, finally, 
$S_{\bar{u}}(\Omega) \simeq \frac{8 \kappa T^{2}}{\pi^{2} \rho D C R} \sum_{K=0}^{\infty} \sum_{N=1}^{\infty} \frac{\xi_{L N}{ }^{2} \exp \left\{-\left[(L+1 / 2)^{2} d^{2} / 2 R_{0}^{2}\right]-\left(\xi_{L N}{ }^{2} b^{2} / 2 R_{0}{ }^{2}\right)\right\}}{\xi_{L N}{ }^{4}+\left(\Omega^{2} R^{4} / D^{2}\right)} \mathcal{R}(L, N)$.

To calculate the sums in a high-frequency approximation we can consider $\mathcal{R}(L, N)=1$. By making the following substitutions: $\quad x=(K+N) / \sqrt{2}, \quad y=(K$ $-N) / \sqrt{2}$, and $\tau_{R}=R^{2} / D$, we use integrals instead of sums:

$$
\begin{aligned}
S_{u} \simeq & \frac{8 \kappa T^{2}}{\pi^{2} \rho C D R} \int_{0}^{\infty} \int_{-x}^{x} \frac{2 \pi^{2} x^{2}}{\pi^{4} x^{4}+\Omega^{2} \tau_{R}^{2}} \\
& \times \exp \left(-\pi^{2} x^{2} b^{2} / R^{2}\right) \exp \left[-(x+y)^{2} / l\right] \mathrm{d} y \mathrm{~d} x \\
\simeq & \frac{8 \kappa T^{2}}{\pi^{2} \rho C D R} \sqrt{\frac{l}{8 \pi}} \int_{0}^{\infty} \frac{t^{2}}{t^{4}+\Omega^{2} \tau_{R}^{2}} \\
& \quad \times \exp \left[-t^{2} b^{2} /\left(2 R^{2}\right)\right] \operatorname{erf}\left(\frac{\sqrt{2} t}{\pi \sqrt{l}}\right) \mathrm{d} t
\end{aligned}
$$

This integral coincides with expression (A5) obtained for infinite media if the error function, which is practically equal to unity for $t>1 / \sqrt{l}$, is ignored. However, spectral densities described by integral (A5) and sum (A15) are different at frequencies for $\Omega<1 / \tau_{R}$, where expression (A15) is finite for $\Omega \rightarrow 0$ (the term with two sums is close to unity).

\section{APPENDIX B: RELATION BETWEEN THE REGULAR CALIBRATION SIGNAL AND THE NOISE SPECTRUM}

To find the link between the amplitude of the harmonic frequency change and the corresponding peak in calculated spectral density and noise spectral density, we consider the Fourier transform for regular and chaotic processes. Let $x(t)$ denote a chaotic process with a correlation function

$$
B(\tau)=\langle x(t) x(t+\tau)\rangle=\int_{-\infty}^{\infty} S^{ \pm}(\Omega) \exp (i \Omega \tau) \frac{\mathrm{d} \Omega}{2 \pi}
$$

where $S^{ \pm}(\Omega)$ stands for double-sided spectral density, which is symmetrical and two times less for positive frequencies than the one-sided $S(\Omega)$. Let $T$ denote the length of the window, $W(t)$, in the time domain, that is, the duration of a set $\{x(t)\}$ (or $\left\{x_{i}\right\}$ in the discrete case) for which the Fourier transform is carried out. Now we consider the transform with square window $w(t)=1$ for $t$ $\in[-T / 2, T / 2]$. The Fourier transform for $x(t)$ is

$$
X_{j}=\int_{-T / 2}^{T / 2} W(t) x(t) \exp \left(-i \Omega_{j} t\right) \mathrm{d} t, \quad \Omega_{j}=\frac{2 \pi j}{T} .
$$

In the procedure of spectrum estimation the spectra $X_{j}(\Omega)$ are calculated for small intervals of data and then averaged, which in our notation corresponds to calculation of the quantity $\left\langle\left|X_{j}\right|^{2}\right\rangle$ :

$$
\begin{aligned}
\left\langle\left|X_{j}\right|^{2}\right\rangle & =\int_{-T / 2}^{T / 2}\left\langle x(t) x\left(t^{\prime}\right)\right\rangle \exp \left[i \omega_{j}\left(t-t^{\prime}\right)\right] \mathrm{d} t \mathrm{~d} t^{\prime} \\
& =\int_{-\infty}^{\infty} S(\omega) \frac{4 \sin ^{2}\left[\left(\omega_{j}-\omega\right)(T / 2)\right]}{\left(\omega_{j}-\omega\right)^{2}} \frac{\mathrm{d} \omega}{2 \pi}
\end{aligned}
$$

Calibrating the peak in our case corresponds to frequency $\omega_{j} \gg T^{-1}$. Then the core in the latter integral in Eq. (B3) has a sharp maximum at $\omega=\omega_{j}$ and $\left\langle\left|X_{j}\right|^{2}\right\rangle$ $=T S\left(\omega_{j}\right)$.

Now let $y(t)=Y_{0} \cos (\Omega t)$ represent a regular harmonic signal. The Fourier transform for such signal for a square window will look like

$$
Y_{s}=\int_{-T / 2}^{T / 2} Y_{0} \cos (\Omega t) \exp \left(-i \Omega_{j} t\right) \mathrm{d} t
$$

leading to $Y_{s j}=Y_{0} T / 2$. Now we compare the results of the spectrum estimation procedure [Eq. (B3)] and the results of the Fourier transform for the harmonic signal:

$$
Y_{s j}{ }^{2}=\frac{Y_{0}{ }^{2} T^{2}}{4} \Leftrightarrow T S^{ \pm}\left(\omega_{j}\right)=\left\langle\left|X_{j}\right|_{s}{ }^{2}\right\rangle .
$$

Here one can easily see the link between the amplitude of the harmonic signal and the corresponding peak in spectral density. If the harmonic signal has the dimension of frequency and the desired spectral density represents relative frequency changes, then the final relation, corresponding to the one-sided spectral density of the calibration spike with a square window in Fourier transform, will be

$$
\sqrt{S_{\delta f / f}} \Leftrightarrow \frac{Y_{0}}{f_{0}} \sqrt{\frac{T}{2}}\left[\frac{1}{\sqrt{\mathrm{Hz}}}\right],
$$

where $f_{0}$ is optical frequency. The same calculations for the Hann window give

$$
\sqrt{S_{\delta f / f}} \Leftrightarrow \frac{Y_{0}}{f_{0}} \sqrt{\frac{T}{3}}\left[\frac{1}{\sqrt{\mathrm{Hz}}}\right]
$$

\section{APPENDIX C: FORMULAS FOR THE IDENTIFICATION OF WGMs}

To identify the parameters of WGMs from photographs we used the following approximation for the half-width of a WGM belt:

$$
\Delta \theta=[2(l+1 / 2-m) / l]^{1 / 2} .
$$


To calculate index $l$ from the radius of a microsphere we found by numerical approximation the following approximate formulas that are valid for $R \gg \lambda$ :

$$
\begin{array}{ll}
q=1: & l=-0.5+t_{l q}-2.287 t_{l q}{ }^{1 / 3}+0.1718 t_{l q}{ }^{-1 / 3}, \\
q=2: & l=-0.5+t_{l q}-4.617 t_{l q}^{1 / 3}+0.6944 t_{l q}{ }^{-1 / 3}, \\
q=3: & l=-0.5+t_{l q}-6.895 t_{l q}^{1 / 3}+1.518 t_{l q}{ }^{-1 / 3} \\
q=4: & l=-0.5+t_{l q}-9.190 t_{l q}^{1 / 3}+2.632 t_{l q}{ }^{-1 / 3}
\end{array}
$$

where

$$
t_{l q}=\frac{2 \pi n_{i} R}{\lambda_{l m q}}+\frac{p n_{i}}{\sqrt{n_{i}^{2}-1}} .
$$

These formulas allow an estimate of values of $l$ and $l$ - $m$ to be obtained from the radius of the microsphere and the width of the belt of the mode that appears as a result of residual surface scattering.

\section{ACKNOWLEDGMENTS}

We are grateful to V. B. Braginsky, whose initial idea to measure thermorefractive noise in microspheres is embodied in this paper. We especially thank S. P. Vyatchanin and F. Ya. Khalili for stimulating and fruitful discussions. This research was partially supported by National Science Foundation grant PHY-0098715 and by President of the Russian Federation grants for young scientists MD208.2003.02 and NS-1318.2003.2.

M. L. Gorodetsky's e-mail address is gorm@hbar.phys.msu.ru.

\section{REFERENCES AND NOTES}

1. A. Abramovici, W. E. Althouse, R. W. P. Drever, Y. Gursel, S. Kawamura, F. J. Raab, D. Shoemaker, L. Sievers, R. E. Srero, K. S. Thorne, R. E. Vogt, R. Weiss, S. E. Whitcomb, and M. E. Zucker, "The project LIGO," Science 256, 325335 (1992)

2. V. B. Braginsky, M. L. Gorodetsky, and S. P. Vyatchanin, "Thermodynamical fluctuations and photo-thermal shot noise in gravitational wave antennae," Phys. Lett. A 264, 1-10 (1999).

3. V. B. Braginsky, M. L. Gorodetsky, and S. P. Vyatchanin, "Thermo-refractive noise in gravitational wave antennae," Phys. Lett. A 271, 303-307 (2000).

4. Y. T. Liu and K. S. Thorne, "Thermoelastic noise and homogeneous thermal noise in finite sized gravitational-wave test masses," Phys. Rev. D 62, 122002 (2000).

5. G. Cagnoli and P. A. Willems, "Effects of nonlinear thermoelastic damping in highly stressed fibers," Phys. Rev. B 65, 174111 (2002).

6. W. H. Glenn, "Noise in interferometric optical systems: An optical Nyquist theorem," IEEE J. Quantum Electron. 25, 1218-1224 (1989).

7. K. H. Wanser, "Fundamental phase noise limit in optical fibres due to temperature fluctuations," Electron. Lett. 28, 53-54 (1992).

8. K. H. Wanser, A. D. Kersey, and A. Dandridge, "Measurement of fundamental thermal phase fluctuations in optical fiber," in International Conference on Integrated Optics and Optical Fiber Communication, Vol. 4 of 1993 OSA Technical Digest Series (Optical Society of America, Washington, D.C., 1993), pp. 255-258.

9. V. B. Braginsky, M. L. Gorodetsky, and V. S. Ilchenko, "Quality-factor and nonlinear properties of optical whispering-gallery modes," Phys. Lett. A 137, 393-397 (1989).

10. M. L. Gorodetsky, V. S. Ilchenko, and A. A. Savchenkov, "Ultimate $Q$ of optical microsphere resonators," Opt. Lett. 21 453-455 (1996).

11. M. L. Gorodetsky and V. S. Ilchenko, "Thermal nonlinear effects in optical whispering-gallery microresonators," Laser Phys. 2, 1004-1009 (1992).

12. M. L. Gorodetsky, "Thermodynamical fluctuations in optical microspheres," in Laser Resonators IV, A. Kudryashov and H. Paxton, eds., Proc. SPIE 4270, 147-153 (2001).

13. M. L. Gorodetsky, S. P. Vyatchanin, and P. A. Willems, unpublished analysis.

14. M. L. Gorodetsky, A. D. Pryamikov, and V. S. Ilchenko, "Rayleigh scattering in high-Q microspheres," J. Opt. Soc. Am. B 17, 1051-1057 (2000).

15. M. L. Gorodetsky and V. S. Ilchenko, "High- $Q$ optical whispering-gallery microresonators: precession approach for spherical mode analysis and emission patterns with prism couplers," Opt. Commun. 113, 133-143 (1994). 